\title{
Red Clump Stars - Further Improved Distance Indicator
}

\author{
P. M. Garnavich \\ University of Notre Dame \\ K. Stanek \\ University of Notre Dame
}

\begin{abstract}
The ideal distance indicator would be a standard candle abundant enough to provide many examples within reach of parallax measurements and sufficiently bright to be seen out to Local Group galaxies. The red clump stars closely match this description. These are the metal rich equivalent of the better known horizontal branch stars, and their brightness dispersion is only $0.2 \mathrm{mag}$ (one sigma) in the Solar neighborhood. Using Hipparcos to calibrate a large, local sample, the red clump method has been used to measure accurate distances to the Galactic center (Paczyński \& Stanek 1998), M31 (Stanek \& Garnavich 1998), LMC (Udalski et al. 1998; Stanek et al. 1998; Udalski 1999) and some clusters in our Galaxy (e.g. 47Tuc: Kaluzny et al. 1998). As with all the distance indicators, the main worry lies in the possible systematics of the method, in particular, the brightness dependence on the stellar metallicity and age. These dependences have come under close scrutiny and, indeed, the population effects on the red clump brightness appear small and calibratable. Perhaps the most controversial result from the red clump method is the estimation of a "short" distance to the Large Magellanic Cloud (Udalski et al. 1998; Stanek, Zaritsky \& Harris 1998; Udalski 2000). This distance to the LMC is shorter by $12 \%$ than the "standard" value, and has very important implications for the Cepheid distance scale and the determination of the Hubble constant.
\end{abstract}

\section{References}

Kaluzny, J. et al. 1998, AJ, 115, 1016

Paczyński, B., \& Stanek, K.Z. 1998, ApJ, 494, L219

Stanek, K., \& Garnavich, P.M. 1998, ApJ, 503, L131

Stanek, K., Zaritsky, D., \& Harris, J. 1998, ApJ, 500, L141

Udalski, A. 2000, ApJ, 531, L25

Udalski, A., et al. 1998, Acta Astr., 48, 1 